\title{
Characterization and Expression Analysis of Genes Encoding Phosphoenolpyruvate Carboxylase and Phosphoenolpyruvate Carboxylase Kinase of Lotus japonicus, a Model Legume
}

\author{
Tomomi Nakagawa, ${ }^{1}$ Tomoko Izumi, ${ }^{2}$ Mari Banba, ${ }^{2}$ Yosuke Umehara, ${ }^{3}$ Hiroshi Kouchi, ${ }^{3}$ Katsura Izui, ${ }^{1,2}$ \\ and Shingo Hata ${ }^{1,2}$ \\ ${ }^{1}$ Laboratory of Plant Physiology, Graduate School of Agriculture, Kyoto University, Sakyo-ku, Kyoto 606-8502, Japan; \\ ${ }^{2}$ Graduate School of Biostudies, Kyoto University, Sakyo-ku, Kyoto 606-8502, Japan; ${ }^{3}$ National Institute of Agrobiological \\ Sciences, Tsukuba, Ibakaki 305-8602, Japan
}

Submitted 1 July 2002. Accepted 3 December 2002.

\begin{abstract}
Phosphoenolpyruvate carboxylases (PEPCs), one form of which in each legume species plays a central role in the carbon metabolism in symbiotic root nodules, are activated through phosphorylation of a conserved residue by a specific protein kinase (PEPC-PK). We characterized the cDNAs for two PEPC isoforms of Lotus japonicus, an amide-translocating legume that forms determinate nodules. One gene encodes a nodule-enhanced form, which is more closely related to the PEPCs in amide-type indeterminate nodules than those in ureide-type determinate nodules. The other gene is expressed in shoots and roots at a low level. Both forms have the putative phosphorylation site, Ser11. We also isolated a cDNA and the corresponding genomic DNA for PEPC-PK of $L$. japonicus. The recombinant PEPC-PK protein expressed in Escherichia coli phosphorylated recombinant maize C4-form PEPC efficiently in vitro. The level of mRNA for PEPC-PK was high in root nodules, and those in shoots and roots were also significant. In situ hybridization revealed that the expression patterns of the transcripts for PEPC and PEPC-PK were similar in mature root nodules, but were different in emerging nodules. When $L$. japonicus seedlings were subjected to prolonged darkness and subsequent illumination, the activity of PEPC-PK and the mRNA levels of both PEPC and PEPC-PK in nodules decreased and then recovered, suggesting that they are regulated according to the amounts of photosynthates transported from shoots.
\end{abstract}

Additional keywords: carbon metabolism, gene expression, symbiotic root nodule.

Phosphoenolpyruvate carboxylases (PEPCs; EC 4.1.1.31) are ubiquitous anaplerotic enzymes in higher plants. Certain molecular species of PEPCs are well known to catalyze the

Corresponding author: S. Hata, Fax: +81 75753 6470;

E-mail: shing@kais.kyoto-u.ac.jp

The first two authors should be considered as joint first authors.

The nucleotide sequence data reported are available in the DDBJ/EMBL/GenBank databases under accession numbers AB092820 (LjPEPC1 cDNA), AB092821 (LjPEPC2 cDNA), AB092818 (LjPEPC$P K$ cDNA), and AB092819 (LjPEPC-PK genomic DNA). photosynthetic $\mathrm{CO}_{2}$ fixation in the leaves of $\mathrm{C} 4$ and Crassulacean acid metabolism (CAM) plants (Chollet et al. 1996; Vidal and Chollet 1997). In the symbiotic nodules of legume roots, PEPCs also play important roles by (i) supplying energy sources such as malate to bacteroids; (ii) supplying the carbon skeleton for the assimilation of fixed nitrogen into amides or ureides; (iii) maintaining the charge balance in xylem sap, which contains amides or ureides; and (iv) recycling $\mathrm{CO}_{2}$ generated through respiration in both plant cells and bacteroids (Deroche and Carrayol 1988; Tajima and Kouchi 1996). Because of these multiple roles, a nodule-enhanced PEPC in each legume is highly expressed in a wide range of nodule cells, including infected and uninfected cells, and cortical cells (Hata et al. 1998; Robinson et al. 1996; Vidal et al. 1986). On the other hand, the activity of bacterial PEPC is not detectable in bacteroids (Kouchi et al. 1988; Miller et al. 1987).

Plant PEPCs are allosteric enzymes that undergo reversible phosphorylation of a conserved Ser near the $\mathrm{N}$ terminus. The phosphorylated enzymes are more active and much less sensitive to malate, a negative effector, while the dephosphorylated forms are less active and less sensitive to glucose-6-phosphate, a positive effector (Chollet et al. 1996; Nimmo 2000; Vidal and Chollet 1997). The regulatory phosphorylation is thought to be mainly controlled by the varying activity of PEPC kinase (also designated as PEPC protein-serine kinase [PEPC-PK]), because the activity of protein phosphatase $2 \mathrm{~A}$, responsible for the dephosphorylation, is rather constant (Carter et al. 1990, 1991). Studies on root nodule PEPCs have been carried out mainly on soybean and alfalfa. The phosphorylation of the nodule PEPCs of these legumes was first demonstrated in vitro by Schuller and Werner (1993) and Vance and associates (Pathirana et al. 1992; Vance et al. 1994), and then in vivo by Chollet and associates (Zhang et al. 1995). cDNA cloning of the nodule-enhanced PEPCs in these leguminous plants also revealed that they have the phosphorylatable Ser residue near the N terminus (Hata et al. 1998; Pathirana et al. 1992). Malate is present in root nodules at millimolar levels (Rosendahl et al. 1990; Vance and Gantt 1992); therefore, PEPCs must be phosphorylated to exert their physiological functions fully.

The PEPC-PK activity in illuminated leaves of $\mathrm{C} 4$ plants is induced by a light-signal transduction cascade (Chollet et al. 1996; Vidal and Chollet 1997). In contrast, the activity in CAM plants is controlled by a circadian oscillator (Nimmo 2000; Taybi et al. 2000). Very recently, it was suggested that a 
phosphoinositide-specific phospholipase $\mathrm{C}$ is involved in the light-signal transduction in C4 and CAM plants (Bakrim et al. 2001; Coursol et al. 2000). As for PEPC-PKs in legume nodules, it has been postulated that perturbation of photosynthate supply from the shoots is responsible for up- and down-regulation of the enzyme activity, the mechanism involved remaining unclear (Zhang and Chollet 1997; Zhang et al. 1995). PEPCPK activity generally is thought to be mainly regulated at the level of gene expression (Chollet et al. 1996; Nimmo 2000; Vidal and Chollet 1997). However, legume genes for PEPCPKs have not been characterized so far, although a partial cDNA for soybean PEPC-PK has been reported (accession number AF203479).

Lotus japonicus is known as a model leguminous plant, mainly because it is diploid with a small genome, self-compatible, and rather easy to transform (Handberg and Stougaard 1992; ImaizumiAnraku et al. 1997; Jiang and Gresshoff 1997). Additionally, an adequate number of expression sequence tags (ESTs) has been reported (Asamizu et al. 2000; Colebatch et al. 2002; Endo et al. 2000; Poulsen and Pødenphant 2002), and a genome sequencing project is in progress. L. japonicus is unique in that it is an amide-translocating legume that forms determinate nodules (Streeter 1991; Takane et al. 2000), whereas most other legumes are either amide-type plants with indeterminate nodules, like alfalfa and pea, or ureide-type plants with determinate nodules, like soybean. L. japonicus may be a better material than soybean for investigating the physiological functions of PEPC in determinate nodules, because PEPC is involved in the

A

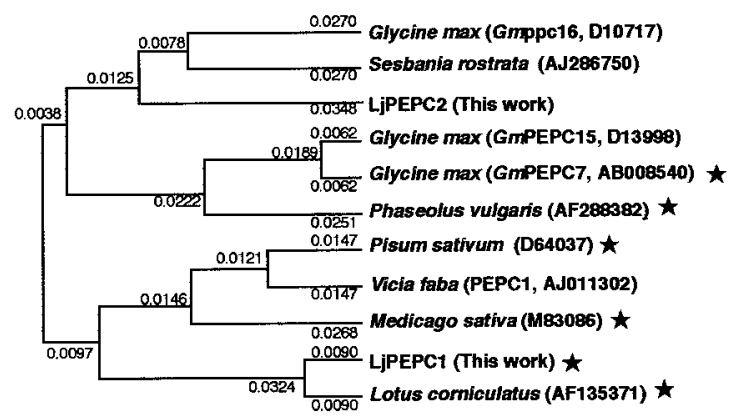

$\mathbf{B}$

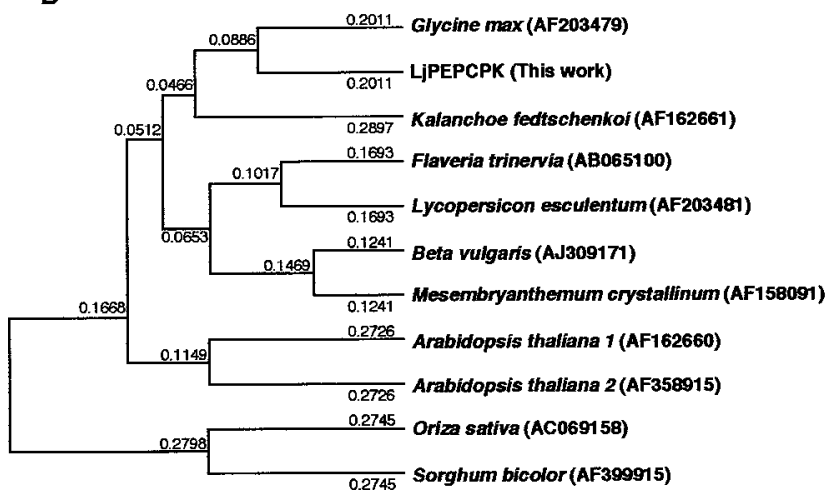

Fig. 1. Phylogenetic relationships of phosphoenolpyruvate carboxylases (PEPCs) and PEPC-protein kinases (PKs) in Lotus japonicus and other plants. A, Phylogenetic tree for the predicted amino acid sequences of full-length cDNAs for legume PEPCs thus far reported. The asterisks denote PEPCs reported to be nodule-enhanced. B, Phylogenetic tree for the predicted amino acid sequences of full-length cDNAs for PEPC-PKs thus far reported. The unweighted pair group method with arithmetic mean tree (Nei 1987) was used to calculate the relative lengths of the branches using Genetyx-Mac software (Genetyx Co., Tokyo). The values near the branches are the branch lengths given as the numbers of amino acid substitutions per site. The database accession numbers also are shown.
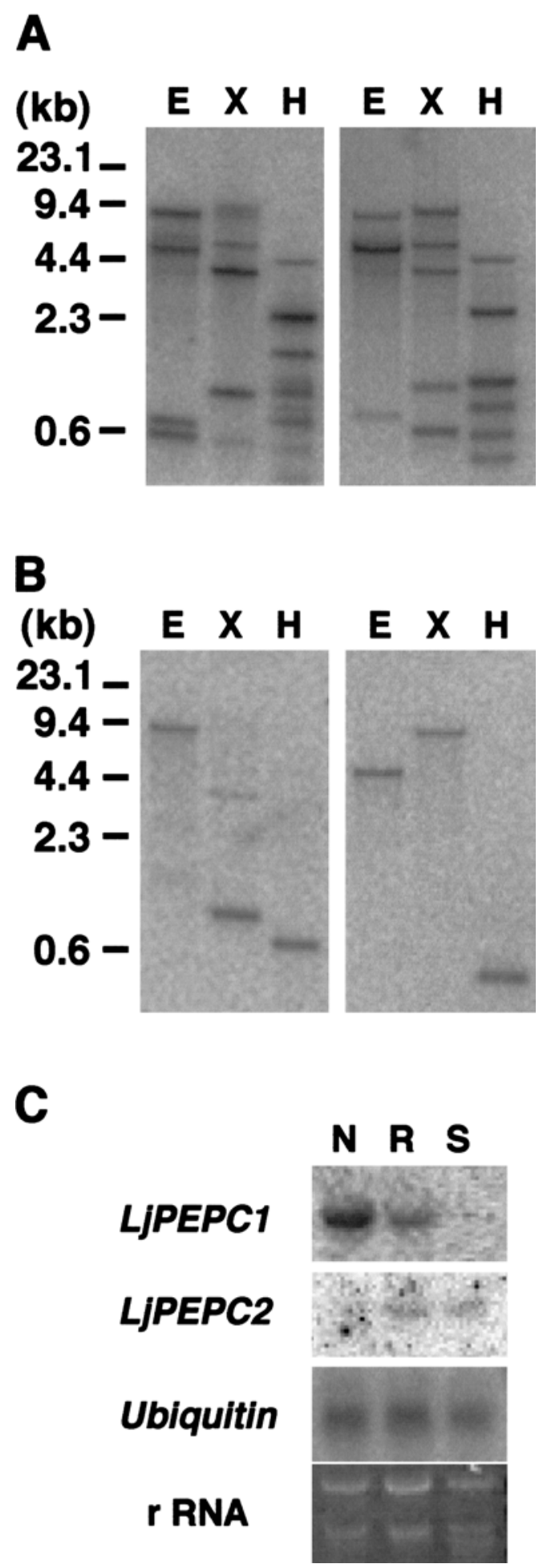

Fig. 2. Blot-hybridization analyses of LjPEPC1 and LjPEPC2. A, Restriction enzyme-digested genomic DNA (10 $\mu \mathrm{g} / \mathrm{lane})$ was separated on $0.8 \%$ agarose gels, transferred to nylon membranes, then probed with the entire LjPEPC1 (left panel) or LjPEPC2 (right panel) cDNA under high-stringency conditions. E, EcoRI; X, XbaI; and H, HindIII. B, The $3^{\prime}$ noncoding region of LjPEPC1 (left panel) or LjPEPC2 (right panel) was used as a probe for genomic Southern analysis. E, EcoRI; X, XbaI; and H, HindIII. C, Total RNA ( $4.5 \mu \mathrm{g} /$ lane $)$ from the indicated parts was separated on a $1 \%$ denaturing agarose gel, transferred to a nylon membrane, hybridized with the $3^{\prime}$ noncoding region of $L j P E P C 1$, then exposed to an imaging plate overnight (top panel). The membrane was stripped, rehybridized with the $3^{\prime}$ noncoding region of $L j P E P C 2$, then exposed to the imaging plate for 3 days (the second panel). The membrane was hybridized again with a cDNA for soybean ubiquitin (Fortin et al. 1988) and then exposed overnight (the third panel). rRNAs stained with ethidium bromide also are shown to confirm the integrity of each RNA preparation (bottom panel). 
production of amides, such as asparagine and glutamine, more directly than in that of ureids. Full-length cDNAs or genomic sequences encoding PEPC-PKs have been reported for several plants (accession numbers: AF399915, AJ309171, AF158091, AF162660, AF162661, AF358915, AF203481, AB065100, and AC069158), making up a family of the smallest protein kinases, but only ones from $\mathrm{C} 4$ and CAM plants have been characterized thus far (Echevarria et al. 2001; Hartwell et al. 1999; Taybi et al. 2000; Tsuchida et al. 2001). In the present study, we isolated the cDNAs for two PEPCs and a PEPC-PK from root nodules of $L$. japonicus, demonstrated the enzyme activity of recombinant PEPC-PK, and examined the expression patterns of these genes.

\section{RESULTS}

cDNA cloning and sequence analyses.

We prepared a cDNA library of mature root nodules of $L$. japonicus as described below. Then, we isolated two molecular species of cDNAs for PEPC isoforms, LjPEPC1 and $L j P E P C 2$, from the library, using the entire GmPEPC7 cDNA for the soybean nodule-enhanced PEPC (Hata et al. 1998) as a probe. Out of $9 \times 10^{5}$ recombinant phages, hundreds of positive clones of $L j P E P C 1$ were detected. On the other hand, only two positive clones of $L j P E P C 2$ were found among $2 \times 10^{5}$ phages. The original LjPEPC2 cDNA was truncated; therefore, its $5^{\prime}$ portion was amplified by polymerase chain reaction (PCR) as described below. Sequence analysis revealed that LjPEPC1 and $L j P E P C 2$ have open reading frames for 961- and 967amino-acid (aa) polypeptides, respectively (data not shown). The cDNAs were 84 and $57 \%$ identical in the coding and $3^{\prime}$ noncoding regions, respectively, at the nucleotide level. The predicted proteins were $90 \%$ identical at the amino acid level. Notably, both the LjPEPC1 and LjPEPC2 proteins had a putative phosphorylation site, Ser11, and the amino acid sequences around this residue were highly conserved among plant PEPCs (data not shown). An extensive database search for L. japonicus ESTs for PEPCs (Asamizu et al. 2000; Colebatch et al. 2002; Endo et al. 2000; Poulsen and Pødenphant 2002) showed that all the sequences for PEPCs were identical to that of either LjPEPC1 or LjPEPC2. However, it is still possible that additional PEPC genes are present in the L. japonicus genome.

To clone the $P E P C$ - $P K$ gene from $L$. japonicus, we prepared redundant oligonucleotide primers based on two unique amino acid clusters showing a high degree of conservation among the published PEPC-PK sequences (Hartwell et al. 1999; Taybi et al. 2000). A DNA fragment, approximately $520 \mathrm{bp}$ in size, was amplified by PCR with the two primers and the cDNA library (data not shown). Sequencing of the PCR product showed that it encodes PEPC-PK as expected. Then, to isolate full-length cDNAs, we screened the above $L$. japonicus nodule cDNA library with the PCR product as a probe. Fourteen positive spots were detected for $7 \times 10^{5}$ clones. Five recombinant phages were picked at random and the cDNA inserts were subcloned into pBluescript II. The longest cDNA, designated as $L j P E P C$ $P K$, had an open reading frame for a 277 -aa polypeptide, the calculated molecular mass of which is $30.727 \mathrm{kDa}$.

Legume PEPCs so far reported form three groups in a phylogenetic tree based on amino acid sequences (Fig. 1A). The LjPEPC1 protein is included in the first group with the noduleenhanced PEPCs of amide-translocating legumes that form indeterminate nodules. The nodule-enhanced PEPCs of ureidetranslocating legumes that form determinate nodules are included in the second group. On the other hand, the LjPEPC2 protein is included in the distantly related third group (Fig. 1A). The predicted LjPEPC-PK protein exhibits the highest amino acid identity with an ortholog of soybean, another leg- ume (Fig. 1B). PEPC-PKs form a family of the smallest protein kinases that lack any regulatory domains (Hanks and Hunter 1995; Hartwell et al. 1999; Taybi et al. 2000), and $L j P E P C-P K$ encodes a typical member of the family.

\section{Genomic organization of $L j P E P C$-PK.}

The $L j P E P C-P K$ gene was amplified by PCR from $L$. japonicus genomic DNA. Despite many positive bands on a genomic Southern blot (discussed below), the amplified DNA was detected as a single band, approximately $1.3 \mathrm{~kb}$ in size, on an agarose gel (data not shown). Sequencing of the gene (AB092819) revealed that there is only one intron, $95 \mathrm{bp}$ in size, at the same position as those of the Kalanchoe PEPC-PK gene (AF162661) and Arabidopsis PEPC-PK2 gene (AY040830).

\section{Blot-hybridization analyses.}

On genomic Southern blotting, the entire cDNAs of LjPEPC1 and LjPEPC2 showed patterns similar to each other, indicating that they cross-hybridize strongly (Fig. 2A). Therefore, we next prepared the $3^{\prime}$ noncoding regions of $L j P E P C l$ and $L j P E P C 2,194 \mathrm{bp}$ and $209 \mathrm{bp}$ in size, respectively. The 3' fragments worked well as isoform-specific probes, because they both gave basically a single band in each lane of the Southern blots (Fig. 2B). On Northern blotting, it was shown that the transcript level of $L j P E P C l$ was highest in root nodules (Fig. 2C). The LjPEPCl mRNA also was detected in noninfected roots at a low level. On the other hand, $L j P E P C 2$ was expressed mainly in roots and shoots at low levels (Fig. 2C). Thus, it was demonstrated that LjPEPCl encodes a noduleenhanced form, as predicted by the phylogenetic tree (Fig. 1A).

Southern blot analysis of $L$. japonicus genomic DNA with the entire $L j P E P C-P K$ cDNA as a probe revealed multiple bands (Fig. 3A). There are two XbaI sites but no EcoRI or HindIII sites in the cDNA. In contrast to the cases of LjPEPCl and $L j P E P C 2$ (Fig. 2B), not only the coding region but also the 3 ' noncoding region of $L j P E P C-P K$ showed multiple bands on the Southern blot (data not shown). Although we could not find

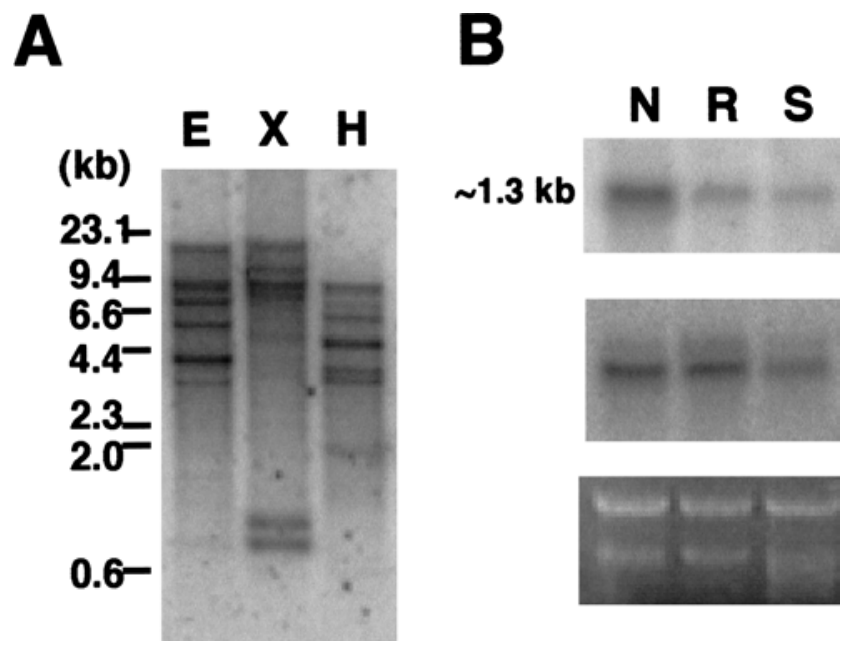

Fig. 3. Blot-hybridization analyses of $L j P E P C-P K$. A, Genomic Southern analysis. Restriction enzyme-digested genomic DNA (10 $\mu \mathrm{g} / \mathrm{lane})$ was separated on a $0.8 \%$ agarose gel, transferred to a nylon membrane, then probed with the entire $L j P E P C-P K$ cDNA under high-stringency conditions. E, EcoRI; X, XbaI; and H, HindIII. B, Northern analysis of the $L j P E P C$ - $P K$ transcript. Total RNA ( $4.5 \mu \mathrm{g} / \mathrm{lane})$ from the indicated parts was separated on a $1 \%$ denaturing agarose gel, transferred to a nylon membrane, then hybridized with the entire $L j P E P C-P K \mathrm{cDNA}$ as a probe (upper panel). The membrane was rehybridized with the soybean ubiquitin probe to confirm the integrity of each RNA preparation (middle panel). rRNAs stained with ethidium bromide also are shown (lower panel). 
proteins closely related to LjPEPC-PK in the above-mentioned L. japonicus entries in databases, other than calcium-dependent protein kinases which are known to be related to PEPC-PKs (Hartwell et al. 1999; Taybi et al. 2000), nonfunctional genes that exhibit accidental sequence similarity to $L j P E P C-P K$ might have cross-hybridized on the Southern blot. Therefore, it is impossible to predict the copy number of PEPC-PK genes in the $L$. japonicus genome. The expression of the LjPEPC-PK transcript, $1.3 \mathrm{~kb}$ in size, was most abundant in nodules (Fig. 3B). In addition, the $L j P E P C-P K$ transcript level also was significant in roots and shoots. It is somewhat strange that a large number of bands and only a single band were detected on Southern and Northern blotting, respectively. Nevertheless, we think that the single band on the Northern blot represents the mRNA level for PEPC-PKs, because transcripts for calcium-dependent protein kinases are longer than $2 \mathrm{~kb}$ (Saijo et al. 1997, 2000).

\section{Confirmation of the enzyme activity} of the recombinant $\mathrm{LjPEPC}-\mathrm{PK}$ protein.

We constructed an expression vector for a fusion protein of a Trx-tag, His-tag, and S-tag (159 amino acids), and LjPEPC-PK (277 amino acids), then introduced it into Escherichia coli BL21 (DE3). The recombinant protein was overexpressed in E. coli with isopropyl-1-thio- $\beta$-galactopyranoside. We also tried to prepare a recombinant LjPEPC1 protein by the same method, but were unsuccessful for an unknown reason (data not shown). Therefore, we checked the phosphorylation activity of LjPEPCPK by incubation with recombinant maize C4-type PEPC and (gamma- ${ }^{32}$ P)ATP. LjPEPC-PK phosphorylated recombinant wild-type maize C4-form PEPC, but not the recombinant S15D

A

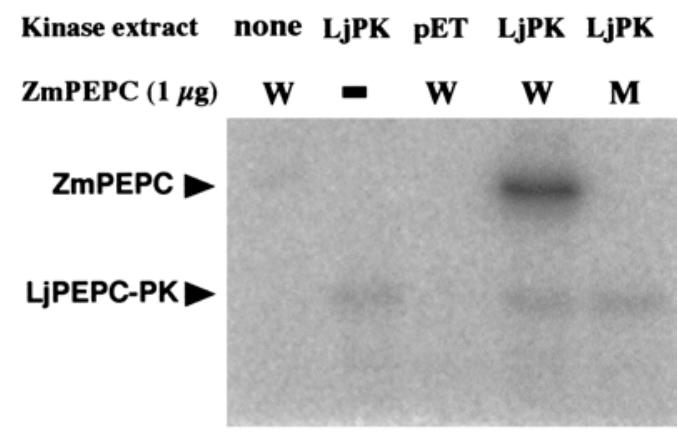

B

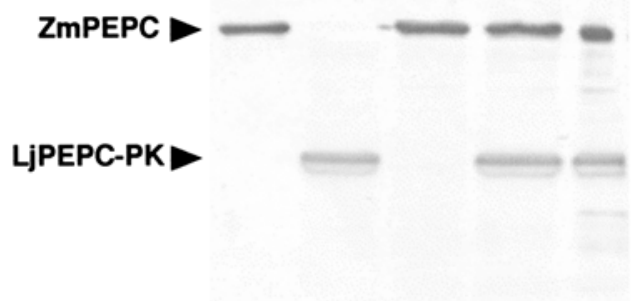

Fig. 4. Confirmation of the specific enzyme activity of the LjPEPC-PK protein. An Escherichia coli extract containing recombinant LjPEPC-PK $(\mathrm{LjPK})$ was incubated with recombinant maize wild-type phosphoenolpyruvate carboxylase (PEPC) (W) or S15D mutant PEPC (M) in the presence of (gamma- $\left.{ }^{32} \mathrm{P}\right)$ ATP. Incubation also was carried out without the extract (none) or with a vector control extract (pET). The reaction products were divided into two portions and separated by $10 \%$ sodium dodecyl sulfate-polyacrylamide gel electrophoresis. A, One gel was subjected to examination with a Bio-imaging analyzer. B, The proteins on the other gel were electrophoretically transferred to an Immobilon membrane, then probed with S-protein-alkaline phosphatase conjugate The positions of recombinant maize PEPC and LjPEPC-PK are indicated on the left. mutant PEPC (Fig. 4) (Ueno et al. 1997). Thus, the phosphorylation of PEPC by LjPEPC-PK was proved in vitro. Faint bands due to autophosphorylation of LjPEPC-PK also were detected. In addition, it was shown that the artificial $\mathrm{N}$ terminal tags of either recombinant PEPCs or PEPC-PKs do not have any effect on the specificity or phosphorylation activity.

\section{In situ localization}

\section{of the LjPEPC1 and LjPEPC-PK transcripts.}

Similar expression patterns of the transcripts of LjPEPC1 and $L j P E P C-P K$ in mature root nodules were observed in an in situ hybridization experiment (Fig. 5A and C). The transcripts were detected in basically all types of nodule and root cells, the signals being most obvious in the vascular bundles of nodules and bacteroid-infected cells. In contrast, the two genes were differentially expressed in rapidly growing nodules (Fig. 5E and G). The transcript of LjPEPCl was localized in the basal portion of the infected zone, whereas that of $L j P E P C-P K$ was detected in the central and top portions of this zone.

\section{Variation in LjPEPC-PK activity.}

To examine variation in LjPEPC-PK activity, root nodules were harvested at the timepoints indicated in Figure 6A. Crude protein extracts were incubated with $\left(\right.$ gamma $\left.-{ }^{32} \mathrm{P}\right) \mathrm{ATP}$ in the presence or absence of exogenous recombinant maize PEPC. The enzyme activity did not change significantly during the photoperiod, although that at the end of the dark period was slightly lower than usual (Fig. 6B, right lanes for samples D8, L8, L16, and D4). Notably, the LjPEPC-PK activity decreased with prolonged darkness and recovered with subsequent illumination (Fig. 6B, right lanes for samples D32 and D-L12). To determine if phospholipase $\mathrm{C}$ is involved in the induction of enzyme activity, illumination was carried out in the presence of an inhibitor, U-73122. However, no more significant inhibition of the $L j P E P C-P K$ expression than that by a control reagent, U-73343, was observed (unpublished data).

Variation in the LjPEPC1 and LjPEPC-PK mRNA levels.

Next, variation in the mRNA levels of LjPEPC1 and $L j P E P C-P K$ in root nodules harvested at the timepoints indicated in Figure 6A was examined (Fig. 7). Although the transcript levels did not change much during the photoperiod, the levels decreased and then recovered with extended darkness and subsequent illumination. Again, U-73122 did not affect the mRNA levels (unpublished data).

\section{DISCUSSION}

In the current study, we have isolated two cDNAs for PEPC isoforms in L. japonicus. It is noteworthy that both forms have the putative phosphorylation site, Ser11. Although we could not find other related sequences in an ample number of ESTs, completion of the L. japonicus genome project is required to determine the total number of genes for PEPCs. One of the $L$. japonicus genes, $L j P E P C 1$, encodes a nodule-enhanced form (Fig. 2C), the transcript of which is abundant in vascular bundles and infected cells of nodules (Fig. 5A). The LjPEPCl mRNA also was detected in noninfected roots at a low level, similar to in the cases of alfalfa and pea (Pathirana et al. 1992; Suganuma et al. 1997). Very recently, Udvardi and associates (Colebatch et al. 2002) found a partial cDNA, LjNEST9G10, that codes for a nodule-induced PEPC as a result of DNA array analysis. LjPEPC1 and LjNEST9G10 are the same, because nucleotides 1,603 to 2,081 of the former are completely identical to nucleotides 2 to 470 of the latter. The other gene, LjPEPC2, was expressed in roots and shoots at low levels, and seemed to encode a housekeeping isoform. 
The present work revealed that nodule-enhanced PEPCs are distinguishable by means of the nitrogen-assimilating compounds (Fig. 1A). This result is in accord with that for uricase in L. japonicus (Takane et al. 2000). However, the result is in contrast with in the case of ENOD40 genes, the genes of legumes being clearly segregated into two distinct groups according to whether the nodules are determinate or indeterminate (Flemetakis et al. 2000). The nodule-enhanced genes involved in metabolism might have been recruited for nodule functions at a different period during evolution from in the case of those involved in the developmental program.

We also isolated a cDNA for PEPC-PK, LjPEPC-PK, which seemed to be the only expressed gene for PEPC-PK in $L$. japonicus. The LjPEPC-PK protein is a typical ortholog of the smallest protein kinases (Fig. 1B), and exhibits high substrate specificity for PEPC in vitro (Fig. 4).

Nodule-enhanced PEPC is a kind of early nodulin, because it is already present before the onset of nitrogen fixation in emerging nodules, but its level dramatically increases at the onset (Hata et al. 1998; Vidal et al. 1986). Expression analysis of genes for nodule-enhanced PEPCs and PEPC-PKs was carried out in detail for the first time. In situ hybridization analysis indicated that the transcript of LjPEPC1 was detected in basically all types of cells in mature nodules, confirming the previous findings at the transcript level (Fedorova et al. 1999; Hata et al. 1998; Kavroulakis et al. 2000; Pathirana et al. 1997) and the protein level (Robinson et al. 1996; Vidal et al. 1986). Moreover, the transcript of $L j P E P C-P K$ was expressed in a similar pattern to that of $L j P E P C 1$ in mature root nodules (Fig. $5 \mathrm{~A}$ and $\mathrm{C}$ ). This result is reasonable because the phosphorylation of PEPC will be required for it to fully perform its multiple functions in nodules. However, the two genes appeared to be differentially expressed in rapidly growing young nodules (Fig. 5E and G). Although the differential expression patterns are intriguing, it remains to be clarified if the two enzyme proteins are localized and exert their functions in the same locations as the respective mRNAs.

Examination of PEPC-PK activity in root nodules revealed that the activity varies only moderately during the day (Fig. 6). This is in contrast to the prominent light-dark response of the leaves of C4 and CAM plants (Bakrim et al. 2001; Echevarria et al. 2001; Taybi et al. 2000; Tsuchida et al. 2001). Nevertheless, when L. japonicus seedlings were subjected to prolonged darkness and subsequent illumination, the activity of LjPEPC-PK in nodules decreased and then recovered (Fig. 6). These results are consistent with the reports of Chollet and associates (Zhang and Chollet 1997; Zhang et al. 1995) and suggest that the LjPEPCPK activity is modulated by the photosynthate supply from shoots. Furthermore, the present work revealed that the mRNA levels of $L j P E P C 1$ and $L j P E P C-P K$ in nodules vary similarly to the PEPC-PK activity (Fig. 7). Thus, these transcript levels also may be regulated by transported photosynthates. However, Western analysis with anti-maize C4-form PEPC antibodies indicated that the protein level of LjPEPC1 in L. japonicus nodules does not change much (unpublished data), consistent with the PEPC level of soybean nodules (Zhang et al. 1995). This shows that variation in the mRNA level does not necessarily accompany that in the protein level. On the other hand, PEPC-PK activity is thought to be mainly regulated at the level of gene expression (Chollet et al. 1996; Nimmo 2000; Vidal and Chollet 1997). The results in Figures 6 and 7 are in line with this view. However, the presence of a protein inhibitor of PEPC-PK in C4 and CAM plants recently was reported (Nimmo et al. 2001). Therefore, the regulation of LjPEPC-PK activity at the protein level should be the subject of intensive investigation in the future. We also performed pharmacological experiments to clarify whether or not phospholipase $\mathrm{C}$ is involved in the $L j P E P C-P K$ gene expression in legume root nodules, as in the signal transduction of $\mathrm{C} 4$ and
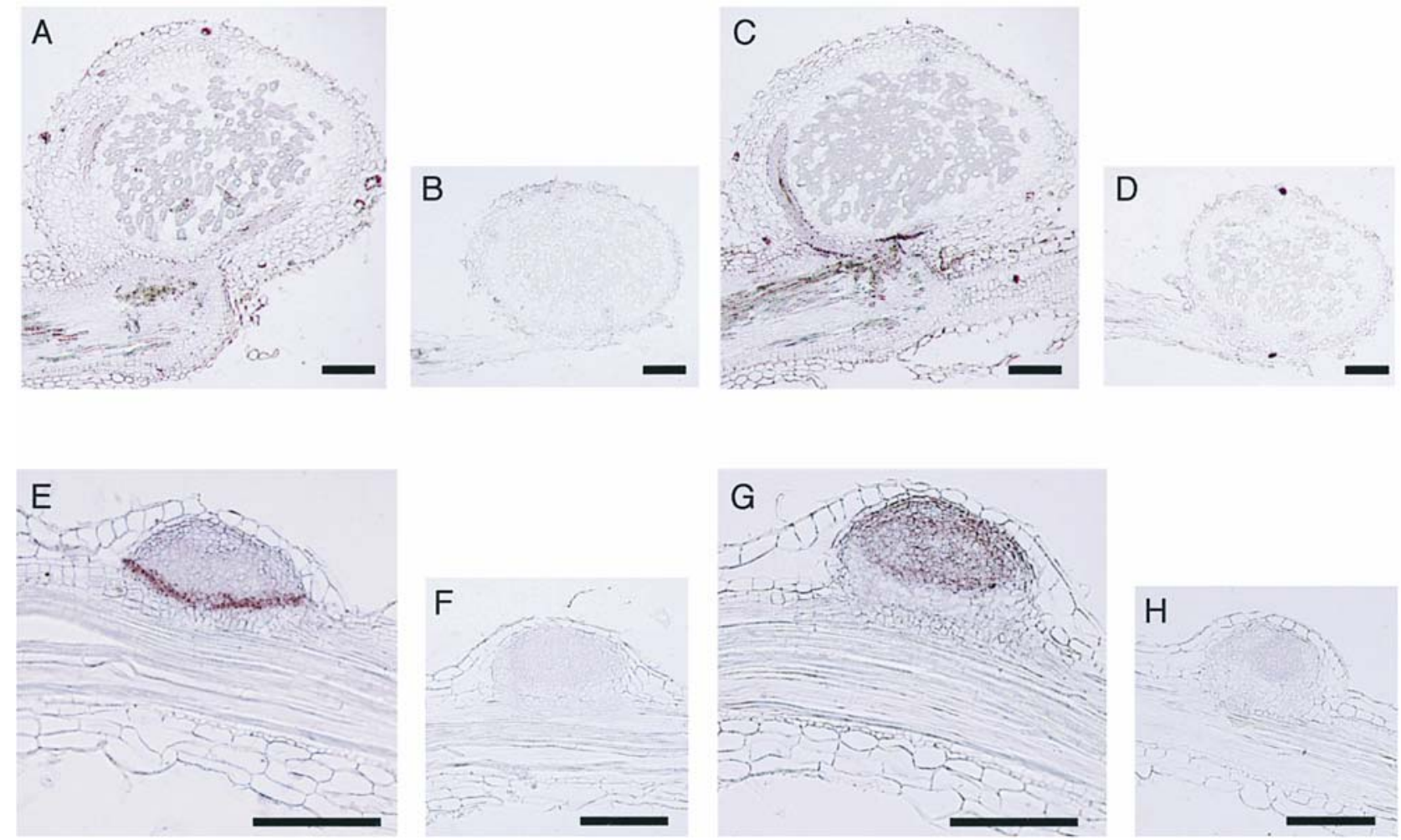

Fig. 5. In situ detection of the $L j P E P C 1$ and $L j P E P C-P K$ transcripts in Lotus japonicus root nodules. A-D, mature nodules; E-H, emerging young nodules. The sections were probed with digoxigenin-labeled antisense RNA prepared from the entire $L j P E P C l \mathrm{cDNA}(\mathrm{A}$ and $\mathrm{E})$ or $L j P E P C-P K \mathrm{cDNA}(\mathrm{C}$ and G). Hybridization signals are visible as a purple or dark blue color. When sense RNA was used as a negative control probe, hybridization, washing, and color development were carried out in side-by-side parallel experiments. No significant hybridization signals were detected $(\mathrm{B}, \mathrm{D}, \mathrm{F}, \mathrm{and} \mathrm{H})$. Bars $=200 \mu \mathrm{m}$. 
A

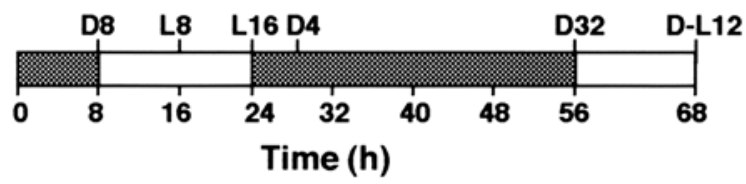

B

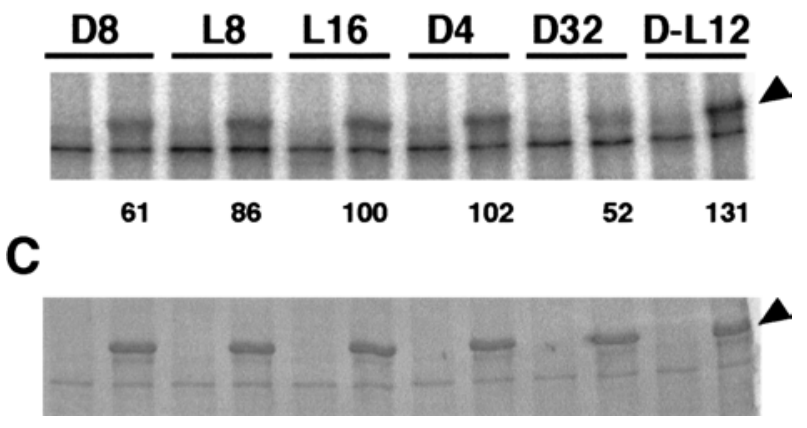

Fig. 6. Circadian variation in the phosphoenolpyruvate carboxylaseprotein kinase (PEPC-PK) activity in Lotus japonicus nodules, and effects of extended darkness and re-illumination. Seedlings were grown for 8 weeks under daily cycles of $16 \mathrm{~h}$ of light and $8 \mathrm{~h}$ of dark. A, Root nodules were harvested at the indicated timepoints. Open and shaded bars indicate the light and dark periods, respectively. The time after initiation of the dark period also is shown. B and $\mathbf{C}$, Each nodule extract (10 $\mu$ g protein) was incubated with $\left(\right.$ gamma $\left.^{32} \mathrm{P}\right)$ ATP in the absence (left lanes) or presence (right lanes) of recombinant maize PEPC (1 $\mu \mathrm{g}$ /incubation), then separated by sodium dodecyl sulfate-polyacrylamide gel electrophoresis. After electrophoresis, the gel was $\mathbf{C}$, stained with Coomassie brilliant blue, then $\mathbf{B}$, subjected to examination with a Bioimaging analyzer for determination of radioactivity. The position of exogenous recombinant maize PEPC is indicated by arrowheads. The relative amounts of ${ }^{32} \mathrm{P}$ incorporated into the exogenous recombinant PEPC also are shown at the bottom of panel B.

CAM plants (Bakrim et al. 2001; Coursol et al. 2000). Although we have not yet obtained positive results (unpublished data), the possibility remains to be examined further.

\section{MATERIALS AND METHODS}

\section{Plant material.}

As described previously (Banba et al. 2001), seeds of Lotus japonicus B-129 "Gifu” were scarified, surface-sterilized, and then grown in sterile vermiculite containing B\&D medium (Broughton and Dilwoth 1971) in double Magenda jars. Mesorhizobium loti "Tono" was inoculated as described (Banba et al. 2001). Unless otherwise stated, plants were grown in a growth chamber at $25^{\circ} \mathrm{C}$ with $60 \%$ humidity under 16-h light $\left(260 \mu \mathrm{Es}^{-1} \mathrm{~m}^{-2}\right)$ and 8-h dark conditions.

\section{Preparation of a cDNA library of $\boldsymbol{L}$. japonicus nodules.}

Double-stranded cDNAs were synthesized with poly $(\mathrm{A})^{+} \mathrm{RNA}$ of mature L. japonicus nodules and oligo(dT), which contains an XhoI site, using a cDNA Synthesis Kit (Amersham Biosciences, Piscataway, NJ, U.S.A.) according to the manufacturer's instructions. After the addition of an EcoRI adaptor, the cDNAs were ligated between the EcoRI and XhoI sites of the lambda ZAP II vector (Stratagene, La Jolla, CA, U.S.A.), and then packaged using a Ready-to-Go Packaging Kit (Amersham Biosciences). The resulting cDNA library contained $3 \times 10^{6}$ independent recombinant phages.

\section{Screening of cDNAs for PEPCs.}

Recombinant phages in the above library were plated with $E$. coli followed by blotting onto Hybond- $\mathrm{N}^{+}$membranes (Amer- sham Biosciences). The entire GmPEPC7 cDNA for soybean nodule-enhanced PEPC (Hata et al. 1998) was radiolabeled with a Megaprime kit and (alpha- ${ }^{32} \mathrm{P}$ )dCTP (Amersham Biosciences) and then used as a probe. The membranes were hybridized at $65^{\circ} \mathrm{C}$ overnight in $6 \times$ SSPE $(1 \times$ SSPE is $0.15 \mathrm{M}$ $\mathrm{NaCl}, 10 \mathrm{mM} \mathrm{NaH} \mathrm{PO}_{4}$, and $1 \mathrm{mM}$ EDTA [pH 7.4]), 5x Denhardt's solution, $0.5 \%$ sodium lauryl sulfate (SDS), and boiled salmon sperm DNA at $200 \mu \mathrm{g} / \mathrm{ml}$ (Sambrook and Russell 2001). Next, the membranes were washed under low stringency conditions with $2 \times$ SSPE and $0.1 \%$ SDS once at room temperature for $10 \mathrm{~min}$ and then twice at $42^{\circ} \mathrm{C}$ for 10 min each. Positive spots were detected by autoradiography. The positive phages were purified and their cDNA inserts were subcloned into pBluescript II. The clone thus isolated was named $L j P E P C 1$.

In the L. japonicus EST database (Asamizu et al. 2000; Colebatch et al. 2002; Endo et al. 2000; Poulsen and Pødenphant 2002), we found another PEPC isoform, named LjPEPC2, and amplified the cDNA fragment by PCR. Using this partial fragment as a probe, we screened longer LjPEPC2 cDNAs in the above library, but the resulting $L j P E P C 2$ cDNA appeared to be truncated by approximately $700 \mathrm{bp}$. One of the LjPEPC2 EST sequences had a putative initiation codon; therefore, we amplified the $5^{\prime}$ portion, $791 \mathrm{bp}$ in size, that includes the region from the library missing by PCR, using a forward primer, 5'TGGATCCGGGTTTGTGAAGTTACAATGGCG-3', and a reverse primer, 5'-CGAATTCAACACGACGCAGAAATTTGGGC-3'. The primers contained a BamHI or EcoRI site to facilitate subsequent cloning.

\section{PCR-based cDNA cloning of LjPEPC-PK.}

Degenerate oligonucleotide primers, 5'-GGAATTCTG(T/C) GA(A/G)GA(A/G)AT(T/C/A)GG-3' and 5'-GCGGATCC (T/ C)TC(T/C/G/A)GG(T/C/G/A)GC(T/C/G/A)AC(A/G)TA(A/G) TA-3', corresponding to the sense sequence of $(\mathrm{Q} / \mathrm{E})(\mathrm{I} / \mathrm{V})$ CEEIG and the antisense sequence of YYVAPEV, respectively, were designed (Tsuchida et al. 2001). They contained EcoRI and BamHI sites to facilitate cloning of the PCR products. PCR was performed for 40 cycles $\left(94^{\circ} \mathrm{C}\right.$ for $1 \mathrm{~min}, 37^{\circ} \mathrm{C}$ for 1 min, and $72^{\circ} \mathrm{C}$ for $1 \mathrm{~min}$ ) with $20 \mu \mathrm{M}$ each of the primers and 1 $\times 10^{7} \mathrm{PFU}$ of the cDNA library. The resulting product, approximately 520 bp in size, was digested with EcoRI and BamHI, subcloned into pBluescript II (Stratagene), then sequenced.

Following the standard protocol described above, $7 \times 10^{5}$ recombinant phages were screened with the radiolabeled PCR product and Hybond- $\mathrm{N}^{+}$membranes. Positive phages were purified and their cDNA inserts were subcloned into pBluescript II for further characterization.

\section{Analysis of $L j P E P C$-PK genomic DNA.}

A genomic DNA fragment was amplified by PCR from the L. japonicus genome using specific primers, 5'-GGCACGAGACAAAACAGAACAATCTAGA-3' and 5'-AGGCCAAAAATACAGAGATCCAA-3', that correspond to the ends of LjPEPC-PK cDNA. The sequence of the resulting fragment was determined and compared to that of the cDNA.

\section{Expression of recombinant LjPEPC-PK.}

The $5^{\prime}$ noncoding region of $L j P E P C-P K$ was removed and an $N c o$ I site was introduced at the initiation codon with the aid of PCR. Then, the product was cloned between the NcoI and XhoI sites of the pET32a vector (Novagen, Madison, WI, U.S.A.). The entire LjPEPC-PK protein (277 aa) fused downstream of a Trx-tag, His-tag, and S-tag (159 aa) was expressed in $E$. coli BL21 (DE3) cells with $0.1 \mathrm{mM}$ isopropyl $\beta$-D-thiogalactopyranoside at $25^{\circ} \mathrm{C}$ for $6 \mathrm{~h}$. The $E$. coli cells were harvested by centrifugation, then suspended in extraction buffer 
comprising $20 \mathrm{mM}$ sodium phosphate ( $\mathrm{pH} 7.5$ ), $0.1 \mathrm{M} \mathrm{NaCl}$, $10 \mathrm{mM}$ imidazole ( $\mathrm{pH} 7.5$ ), $15 \%$ ethylene glycol, $14 \mathrm{mM}$ mercaptoethanol, and $1 \mathrm{mM}$ phenylmethylsulfonyl fluoride. After the cells had been disrupted with a French press and then centrifuged, the supernatant was used for the kinase assay.

In vitro assaying of recombinant $\mathrm{LjPEPC}$-PK activity.

The phosphorylation reaction was carried out at $30^{\circ} \mathrm{C}$ for 15 min in a reaction mixture consisting of $0.1 \mathrm{mM}$ (gamma$\left.{ }^{32} \mathrm{P}\right)$ ATP $(0.5 \mu \mathrm{Ci}), 50 \mathrm{mM}$ Tris- $\mathrm{HCl}(\mathrm{pH} 8.0), 5 \mathrm{mM} \mathrm{MgCl}{ }_{2}, 1$ $\mu \mathrm{g}$ purified recombinant maize PEPC protein with a Trx-tag, His-tag, and S-tag at its N-terminus, and $25 \mu \mathrm{g}$ of a crude extract of recombinant LjPEPC-PK. The reaction products were divided into two portions and then separated by $10 \%$ SDSpolyacrylamide gel electrophoresis (PAGE). One gel was subjected to examination with a BAS2000 bio-imaging analyzer (Fujifilm, Tokyo). The proteins on the other gel were electrophoretically transferred to an Immobilon Transfer Membrane (Millipore, Bedford, MA, U.S.A.). The S-Tagged proteins were detected with a S-Tag System (Novagen) according to the manufacturer's protocol.

\section{Genomic Southern blot analyses.}

Genomic DNA $(10 \mu \mathrm{g})$ from $L$. japonicus was digested with EcoRI, XbaI, or HindIII, separated by electrophoresis on a $0.8 \%$ agarose gel, then transferred to a Hybond- $\mathrm{N}^{+}$membrane. The membrane was hybridized with the entire cDNAs or the PCR-generated $3^{\prime}$ noncoding regions of LjPEPCl and LjPEPC2. Forward and reverse primer pairs (5'-TTGAGTTGCCACCTCCTTTA-3' and 5'-TTGACTCATTTGTGTGGTAGCTGCCT-3', 5'-TGGTTAAATCTGAGAGATTCTACT-3' and 5'-TAATCAGTTGCATACTGGCATTA-3', and 5' -TGAGAAAACTGTGGAGGGTT-3' and 5'-AGGCCAAAAATACAGAGATCCAA-3') were used for amplification of the $3^{\prime}$ noncoding regions of LjPEPC1 (194 bp in size), LjPEPC2 (209 bp in size), and LjPEPC-PK (382 bp in size), respectively.

\section{Northern analyses.}

RNA blot-hybridization analysis was carried out as described previously (Hata et al. 1998) using $4.5 \mu \mathrm{g}$ of total RNA/lane and the ${ }^{32} \mathrm{P}$-labeled probes described above.

\section{In situ hybridization.}

In situ hybridization of PEPC and PEPC-PK transcripts in $L$. japonicus nodules was carried out as described (Kouchi and Hata 1993). In brief, mature and young nodules were harvested with adjacent roots, immediately fixed, dehydrated through a graded ethanol series, and embedded in paraffin. Microtome sections (10 $\mu \mathrm{m}$ thick) were hybridized with digoxygenin-labeled antisense and sense probes at $50^{\circ} \mathrm{C}$ for $16 \mathrm{~h}$, after which successive washings were performed. The probes were synthesized from the entire cDNAs of $L j P E P C-P K$ and $L j P E P C 1$.

\section{Treatment of $L$. japonicus plants}

with prolonged darkness or metabolic inhibitors.

Dark-treatment of seedlings was initiated at the beginning of a regular night. For pharmacological experiments, dark-treated seedlings were transferred to a nutrient solution containing 15 $\mu \mathrm{M} \mathrm{U}-73122$ or U-73343, then illuminated for $12 \mathrm{~h}$.

\section{Preparation of crude nodule extracts.}

Nodules $(0.2 \mathrm{~g})$ harvested at various timepoints were homogenized with a mortar and pestle in liquid nitrogen, then dissolved in $500 \mu \mathrm{l}$ of buffer A (100 mM Tris- $\mathrm{HCl}$ [pH 8.0], 10 $\mathrm{mM} \mathrm{MgCl}_{2}, 1 \mathrm{mM}$ EDTA, 5\% [wt/vol] glycerol, $15 \mathrm{mM} \mathrm{2-}$ mercaptoethanol, $2 \%$ [wt/vol] insoluble polyvinyl-polypyrrolidone, and $1 \mathrm{mM}$ phenylmethylsulfonyl fluoride). After cen-
D8 $\quad$ L8 L16 $\quad$ D4 D32 D-L12

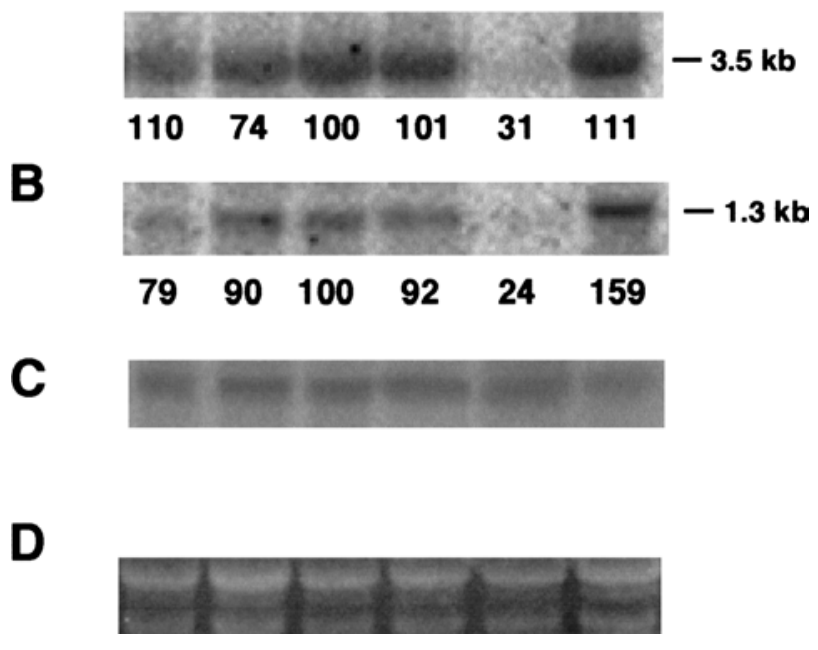

Fig. 7. Circadian variation in the mRNA levels of $L j P E P C 1$ and $L j P E P C$ $P K$, and the effects of extended darkness and re-illumination. Total RNA was extracted from root nodules harvested at various timepoints. RNA preparations ( $4.5 \mu \mathrm{g} / \mathrm{lane})$ were subjected to Northern analyses with entire A, LjPEPC1 cDNA and B, LjPEPC-PK cDNA as probes. The relative radioactivity (averages of three separate experiments) is shown at the bottom of each panel. The membrane was rehybridized with $\mathbf{C}$, the soybean ubiquitin probe. D, rRNAs stained with ethidium bromide also are shown.

trifugation for $30 \mathrm{~min}$ at $50,000 \times g$ at $4^{\circ} \mathrm{C}$, the supernatant was desalted on a Sephadex G-25 column (Amersham Biosciences) preequilibriated with buffer A. The resulting crude extract was used for the kinase assay and Western analysis.

\section{Western analysis of the LjPEPC1 protein.}

A crude nodule extract was separated by $7.5 \%$ SDS-PAGE. After electrophoresis, the proteins were electrophoretically transferred to an Immobilon membrane. Immunoblot analysis with antisera against maize C4-form PEPC was carried out as described previously (Ueno et al. 2000).

\section{Assaying of LjPEPC-PK activity in nodule extracts.}

The phosphorylation reaction was carried out at $30^{\circ} \mathrm{C}$ for 30 min in a reaction mixture consisting of $0.1 \mathrm{mM}$ (gamma$\left.{ }^{32} \mathrm{P}\right)$ ATP $(0.5 \mu \mathrm{Ci}), 50 \mathrm{mM}$ Tris- $\mathrm{HCl}(\mathrm{pH} 8.0), 5 \mathrm{mM} \mathrm{MgCl} 2,1$ mM EGTA, $1 \mathrm{mM}$ DTT, $1 \mu \mathrm{M}$ ocadic acid, $1 \mu \mathrm{g}$ of purified recombinant maize PEPC protein, and $25 \mu \mathrm{g}$ of crude nodule extract. After the reaction, a sample was subjected to $7.5 \%$ SDSPAGE, and then the radioactive bands on the gel were visualized with a BAS2000 imaging analyzer.

\section{ACKNOWLEDGMENTS}

We thank Y. Tsuchida and K. Oshima for the instructions regarding the in vitro phosphorylation assay and Western blotting. This work was supported in part by Special Coordination Funds for Promoting Science and Technology from the Ministry of Education, Culture, Sports, Science, and Technology of the Japanese Government, and a research grant from the Takeda Science Foundation. M. Banba was the recipient of a Research Fellowship for Young Scientists from the Japan Society for the Promotion of Science.

\section{LITERATURE CITED}

Bakrim, N., Brulfert, J., Vidal, J., and Chollet, R. 2001. Phosphoenolpyruvate carboxylase kinase is controlled by a similar signaling cascade in CAM and C4 plants. Biochem. Biophys. Res. Commun. 286:11581162

Banba, M., Siddique, A.-B. M., Kouchi, H., Izui, K., and Hata, S. 2001. Lotus japonicus forms early senescent root nodules with Rhizobium etli. Mol. Plant-Microbe Interact. 14:173-180. 
Broughton, W. J., and Dilwoth, M. J. 1971. Control of leghemoglobin synthesis in snake beans. Biochem. J. 125:1075-1080.

Carter, P. J., Nimmo, H. G., Fewson, C. A., and Wilkins, M. B. 1990 Bryophyllum fedtschenkoi protein phosphatase 2A can dephosphorylate phosphoenolpyruvate carboxylase. FEBS (Fed. Eur. Biochem. Soc.) Lett. 263:233-236.

Carter, P. J., Nimmo, H. G., Fewson, C. A., and Wilkins, M. B. 1991. Circadian rhythms in the activity of a plant protein kinase. EMBO J. 10:2063-2068.

Chollet, R., Vidal, J., and O'Leary, M. H. 1996. Phosphoenolpyruvate carboxylase: a ubiquitous, highly regulated enzyme in plants. Annu. Rev. Plant Physiol. Plant Mol. Biol. 47:273-298

Colebatch, G., Kloska, S., Trevaskis, B., Freund, S., Altmann, T., and Udvardi, M. K. 2002. Novel aspects of symbiotic nitrogen fixation uncovered by transcript profiling with cDNA arrays. Mol. Plant-Microbe Interact. 15:411-420.

Coursol, S., Giglioli-Guivarc'h, N., Vidal, J., and Pierre, J.-N. 2000. An increase in phosphoinositide-specific phospholipase $\mathrm{C}$ activity precedes induction of $\mathrm{C} 4$ phosphoenolpyruvate carboxylase phosphorylation in illuminated and $\mathrm{NH}_{4} \mathrm{Cl}$-treated protoplasts from Digitaria sanguinalis. Plant J. 23:497-506.

Deroche, M. E., and Carrayol, E. 1988. Nodule phosphoenolpyruvate carboxylase: a review. Physiol. Plant. 74:775-782.

Echevarria, C., Garcia-Maurino, S., Alvarez, R., Soler, A., and Vidal, J. 2001. Salt stress increases the $\mathrm{Ca}^{2+}$-independent phosphoenolpyruvate carboxylase kinase activity in Sorghum leaves. Planta 214:283-287.

Endo, M., Kokubun, T., Takahata, Y., Higashitani, A., Tabata, S., and Watanabe, M. 2000. Analysis of expressed sequence tags of flower buds in Lotus japonicus. DNA Res. 7:213-216.

Fedorova, M., Tikhonovich, I. A., and Vance, C. P. 1999. Expression of $\mathrm{C}$-assimilating enzymes in pea (Pisum sativum L.) root nodules: in situ localization in effective nodules. Plant Cell Environ. 22:1249-1262.

Flemetakis, E., Kavroulakis, N., Quaedvlieg, N. E. M., Spaink, H. P. Dimou, M., Roussis, A., and Katinakis, P. 2000. Lotus japonicus contains two distinct ENOD40 genes that are expressed in symbiotic, nonsymbiotic, and embryonic tissues. Mol. Plant-Microbe Interact. 9:987994

Fortin, M. G., Purohit, S. K., and Verma, D. P. S. 1988. The primary structure of soybean (Glycine max) ubiquitin is identical to other plant ubiquitins. Nucleic Acids Res. 16:11377.

Handberg, K., and Stougaard, J. 1992. Lotus japonicus, an autogamous, diploid legume species for classical and molecular genetics. Plant $\mathrm{J}$. 2:487-496.

Hanks, S. K., and Hunter, T. 1995. The eukaryotic protein kinase superfamily: kinase (catalytic) domain structure and classification. FASEB J. 9:576-596.

Hartwell, J., Gill, A., Nimmo, G. A., Wilkins, M. B., Jenkins, G. I., and Nimmo, H. G. 1999. Phosphoenolpyruvate carboxylase kinase is a novel protein kinase regulated at the level of expression. Plant $\mathbf{J}$. 20:333-342.

Hata, S., Izui, K., and Kouchi, H. 1998. Expression of a soybean noduleenhanced phosphoenolpyruvate carboxylase gene that shows striking similarity to another gene for a house-keeping isoform. Plant J. 13:267-273.

Imaizumi-Anraku, H., Kawaguchi, M., Koiwa, H., Akao, S., and Syono, K. 1997. Two ineffective nodulating mutants of Lotus japonicus: different phenotypes caused by the blockage of endocytotic bacterial release and nodule maturation. Plant Cell Physiol. 38:871-881.

Jiang, Q., and Gresshoff, P. M. 1997. Classical and molecular genetics of the model legume Lotus japonicus. Mol. Plant-Microbe Interact. 10:59-68.

Kavroulakis, N., Flemetakis, E., Aivalakis, G., and Katinakis, P. 2000. Carbon metabolism in developing soybean root nodules: the role of carbonic anhydrase. Mol. Plant-Microbe Interact. 13:14-22.

Kouchi, H., Fukai, K., Katagiri, H., Minamisawa, K., and Tajima, S. 1988. Isolation and enzymological characterization of infected and uninfected cell protoplasts from root nodules of Glycine max. Physiol. Plant. 73:327-334.

Kouchi, H., and Hata, S. 1993. Isolation and characterization of novel nodulin cDNAs representing genes expressed at early stages of soybean nodule development. Mol. Gen. Genet. 238:106-119.

Miller, S. S., Boylan, K. L. M., and Vance, C. P. 1987. Alfalfa root nodule carbon dioxide fixation. Immunological studies of nodule phosphoenolpyruvate carboxylase. Plant Physiol. 84:501-508

Nei, M. 1987. Molecular Evolutionary Genetics. Columbia University Press, New York

Nimmo, G. A., Wilkins, M. B., and Nimmo, H. G. 2001. Partial purification and characterization of a protein inhibitor of phosphoenolpyruvate carboxylase kinase. Planta 213:250-257.

Nimmo, H. G. 2000. The regulation of phosphoenolpyruvate carboxylase in CAM plants. Trends Plant Sci. 5:75-80.

Pathirana, M. S., Samac, D. A., Roeven, R., Yoshioka, H., Vance, C. P. and Gantt, S. 1997. Analyses of phosphoenolpyruvate carboxylase gene structure and expression in alfalfa nodules. Plant J. 12:293-304.

Pathirana, S. M., Vance, C. P., Miller, S. S., and Gantt, J. S. 1992. Alfalfa root nodule phosphoenolpyruvate carboxylase: characterization of the cDNA and expression in effective and plant-controlled ineffective nodules. Plant Mol. Biol. 20:437-450.

Poulsen, C., and Pødenphant, L. 2002. Expressed sequence tags from roots and nodule primordia of Lotus japonicus infected with Mesorhizobium loti. Mol. Plant-Microbe Interact. 15:376-379.

Robinson, D. L., Pathirana, S. M., Gantt, J. S., and Vance, C. P. 1996. Immunogold localization of nodule-enhanced phosphoenolpyruvate carboxylase in alfalfa. Plant Cell Environ. 19:602-608.

Rosendahl, L., Vance, C. P., and Pederson, W. B. 1990. Products of dark $\mathrm{CO}_{2}$ fixation in pea root nodules support bacteroid metabolism. Plant Physiol. 93:12-19.

Saijo, Y., Hata, S., Kyozuka, J., Shimamoto, K, and Izui, K. 2000. Overexpression of a single $\mathrm{Ca}^{2+}$-dependent protein kinase confers both cold and salt/drought tolerance on rice plants. Plant J. 23:319-327.

Saijo, Y., Hata, S., Sheen, J., and Izui, K. 1997. CDNA cloning and prokaryotic expression of maize calcium-dependent protein kinases. Biochim. Biophys. Acta 1350:109-114.

Sambrook, J., and Russell, D. W. 2001. Molecular Cloning: A Laboratory Manual, 3rd ed. Cold Spring Harbor Laboratory Press, Cold Spring Harbor, NY.

Schuller, K. A., and Werner, D. 1993. Phosphorylation of soybean (Glycine $\max$ L.) nodule phosphoenolpyruvate carboxylase in vitro decreases sensitivity to inhibition by L-malate. Plant Physiol. 101:12671273

Streeter, J. G. 1991. Transport and metabolism of carbon and nitrogen in legume nodules. Adv. Bot. Res. 18:129-187.

Suganuma, N., Okada, Y., and Kanayama, Y. 1997. Isolation of a cDNA for nodule-enhanced phosphoenolpyruvate carboxylase from pea and its expression in effective and plant-determined ineffective pea nodules. J. Exp. Bot. 48:1165-1173.

Tajima, S., and Kouchi, H. 1996. Metabolism and compartmentation of carbon and nitrogen in legume nodules. Pages 27-60 in: Plant-Microbe Interactions. G. Stacey, and N. Keen, eds. Chapman \& Hall, New York.

Takane, K., Tajima, S., and Kouchi, H. 2000. Structural and expression analysis of uricase mRNA from Lotus japonicus. Mol. Plant-Microbe Interact. 13:1156-1160.

Taybi, T., Patil, S., Chollet, R., and Cushman, J. C. 2000. A minimal serine/threonine protein kinase circadianly regulate phosphoenolpyruvate carboxylase activity in Crassulacean acid metabolism-induced leaves of the common ice plant. Plant Physiol. 123:1471-1481.

Tsuchida, Y., Furumoto, T., Izumida, A., Hata, S., and Izui, K. 2001. Phosphoenolpyruvate carboxylase kinase involved in $\mathrm{C} 4$ photosynthesis in Flaveria trinervia: cDNA cloning and characterization. FEBS (Fed. Eur. Biochem. Soc.) Lett. 507:318-322.

Ueno, Y., Hata, S., and Izui, K. 1997. Regulatory phosphorylation of plant phosphoenolpyruvate carboxylase: role of a conserved basic residue upstream of the phosphorylation site. FEBS (Fed. Eur. Biochem. Soc.) Lett. 417:57-60.

Ueno, Y., Imanari, E., Emura, J., Yoshizawa-Kumagaye, K., Nakajima, K., Inami, K., Shiba, T, Sakakibara, H., Sugiyama, T., and Izui, K. 2000. Immunological analysis of the phosphorylation state of maize C4-form phosphoenolpyruvate carboxylase with specific antibodies raised against a synthetic phosphorylated peptide. Plant J. 21:17-26.

Vance, C. P., and Gantt, J. S. 1992. Control of nitrogen and carbon metabolism in root nodules. Physiol. Plant. 85:266-274.

Vance, C. P., Gregerson, R. G., Robinson, D. L., Miller, S. S., and Gantt, J. S. 1994. Primary assimilation of nitrogen in alfalfa nodules: molecular features of the enzymes involved. Plant Sci. 101:51-64.

Vidal, J., and Chollet, R. 1997. Regulatory phosphorylation of C4 PEP carboxylase. Trends Plant Sci. 2:230-237.

Vidal, J., Nguyen, J., Perrot-Rechenmann, C., and Gadal, P. 1986. Phosphoenolpyruvate carboxylase in soybean root nodules: an immunochemical study. Planta 169:198-201.

Zhang, X.-Q., and Chollet, R. 1997. Phosphoenolpyruvate carboxylase protein kinase from soybean nodules: partial purification, characterization, and up/down-regulation by photosynthate supply from the shoots. Arch. Biochem. Biophys. 343:260-268.

Zhang, X.-Q., Li, B., and Chollet, R. 1995. In vivo regulatory phosphorylation of soybean nodule phosphoenolpyruvate carboxylase. Plan Physiol. 108:1561-1568. 